\title{
Lista dos Exemplares Tipos de Ceratopogonidae (Diptera, Nematocera) Depositados na Coleção Entomológica do Instituto Oswaldo Cruz, Rio de Janeiro, Brasil
}

\author{
ML Felippe-Bauer ${ }^{+}$, SJ de Oliveira* \\ Laboratório de Díptera *Coleção Entomológica, Departamento de Entomologia, Instituto Oswaldo Cruz-Fiocruz, \\ Av. Brasil 4365, 21045-900, Rio de Janeiro, RJ, Brasil
}

List of the Type Species of Ceratopogonidae (Diptera, Nematocera) Deposited in the Entomological Collection of Instituto Oswaldo Cruz, Rio de Janeiro, Brazil - A list of all type specimens of the Family Ceratopogonidae, present in the Entomological Collection of Instituto Oswaldo Cruz, Rio de Janeiro, RJ, Brazil is presented. This list includes the genera Bahiahelea, Culicoides, Dasyhelea, Downeshelea, Forcipomyia, Leptoconops, Mallochohelea, Monohelea, Neobezzia, Palpomyia and Sphaerohelea.

Key words: Diptera - Ceratopogonidae - type species - Entomological Collection - Instituto Oswaldo Cruz Rio de Janeiro - Brazil

\begin{abstract}
Adolpho Lutz (1903) em uma "Nota preliminar sobre os insectos sugadores de sangue observados nos Estados de S. Paulo e Rio de Janeiro", diz que "Os dipteros conhecidos pelos nomes de polvora, maruim ou mosquitinhos de mangue são nematoceros e pertencem ao genero ceratopogon. Possuimos duas especies não determinadas, das quaes a maior é encontrada principalmente na zona marítima, enquanto que a menor frequenta as matas humidas. Ambas são muito avidas de sangue. Julgamos provavel que existam mais espécies em condições analogas".
\end{abstract}

DW Coquillett (1904) descreveu o Ceratopogon guttatus (hoje Culicoides guttatus), primeira espécie de um ceratopogonídeo descrito no Brasil, baseado em três fêmeas colecionadas por Lutz em São Paulo, SP.

Antonio Emilio Goeldi (1905) no seu conhecido trabalho "Os mosquitos no Pará", descreveu um gênero novo e uma espécie nova, Haematomyidium paraense (hoje $C$. paraensis). Cansado de esperar a identificação de material brasileiro enviado para pesquisadores estrangeiros, fez um desabafo: "Resolvi na última hora denominar e descrever summariamente o "miruim" paraense para acabar finalmente com esta afflictiva situação de incerteza que já por demais tempo dura acerca da sua posi-

\footnotetext{
${ }^{+}$Corresponding author. Fax: +55-21-2573.4468. E-mail: mlfbauer@gene.dbbm.fiocruz.br

Received 5 April 2001

Accepted 11 July 2001
}

ção systematica". Goeldi, na verdade, estava descrevendo a espécie mais importante da família Ceratopogonidae no Brasil, pois, sabe-se hoje, é vetora de filarioses e viroses que atingem homens e animais. Curioso é que Goeldi, sem conhecer o trabalho de Coquillett discorda de Lutz quanto à ocorrência do gênero Ceratopogon no Brasil, afirmando: "Tanto quanto sei ainda não há especie alguma de Ceratopogon até hoje descripta no Brazil".

Os tipos dessas duas primeiras espécies descritas para o Brasil foram perdidos, mas Fox (1958) e Wirth e Felippe-Bauer (1989) designaram lectótipo e neótipo, respectivamente, para $C$. guttatus e paraensis que se encontram depositados na Coleção Entomológica do Instituto Oswaldo Cruz (IOC).

Lutz, depois de trabalhar muitos anos no Laboratório Bacteriológico de São Paulo (hoje Instituto Adolpho Lutz), passou a trabalhar no IOC, a partir de 1908. Com ele começou a tradição de se ter no Instituto especialistas trabalhando em Ceratopogonidae. Assim, Lutz publicou em 1912, $1913 \mathrm{e}$ 1914 três trabalhos que resultaram na descrição de 15 espécies novas, dentre elas o C. maruim, colecionado nos mangues de Manguinhos. Angelo M da Costa Lima (1928) descreveu a espécie Forcipomyia obesa e, em 1937, descreveu mais três espécies: C. flavivenulus, C. lutzi e Pterobosca macfiei [hoje Forcipomyia (Pterobosca) macfiei].

Cesar Pinto (1930) no seu livro Arthropodes Parasitos e Transmissores de Doenças, dedica um capítulo às "Ceratopogoninas hematophagas" listando 14 espécies brasileiras.

O estudo taxonômico dos Ceratopogonidae só foi retomado, no IOC, 40 anos depois por Omar 
Tavares e colaboradores que descreveram 14 espécies entre os anos de 1978 a 1980. Este estudo foi interrompido com a morte prematura de Tavares. A partir de 1987, ML Felippe-Bauer e colaboradores, vêm dando continuidade aos estudos dos Ceratopogonidae não apenas do Brasil, mas dos da região neotrópica como um todo; até o ano 2000 foram descritas 18 espécies.

A Coleção Entomológica do IOC abriga 2 milhões de exemplares das mais diversas ordens de insetos, incluindo tipos dos acervos trabalhados por pesquisadores como A Lutz, A Neiva, AM da Costa Lima, C Pinto, H de Souza Lopes, FL Werneck, O Mangabeira Filho, JF Zikán, H Lent, J Jurberg, R Carcavallo, dentre outros. Seguindo as recomendações do Código Internacional de Nomenclatura Zoológica que estabelece que toda a Instituição que contém espécimens tipos deve publicar lista dos tipos ali depositados, foram feitas listas de Hemiptera-Triatominae (Gonçalves et al. 1993, Rocha et al. 1998), Diptera-Culicidae (Marchon-Silva et al. 1996), Hymenoptera (Ferraz 1997) e de Anoplura (Cardozo de Almeida et al. 1999).

No presente trabalho é fornecida uma lista dos espécimens tipos de Ceratopogonidae depositados na Coleção Entomológica do IOC.

Os espécimens tipos estão depositados nas seções "Coleção Geral" e "Coleção Costa Lima". Os exemplares incluídos na Coleção Costa Lima estão devidamente indicados no texto; alguns espécimens apresentam, além do número da lâmina, uma numeração protocolar.

A maior parte da coleção se encontra montada em lâmina e os espécimens tipos, montados em alfinete ou conservados em álcool, estão devidamente indicados no texto.

Os espécimens tipos pertencem a 102 espécies, que estão incluídas em 11 gêneros. Deste material, estão depositados na Coleção holótipos, alótipos, parátipos, lectótipos, neótipos, cótipos e exemplares pertencentes à série típica original.

Os nomes científicos, ao nível de subfamília, tribo, gênero e espécie, estão listados em ordem alfabética.

Ao nome específico segue-se o nome do autor, data da publicação, dados relacionados com o exemplar tipo e informações adicionais, quando existentes.

No caso de novas combinações, o nome genérico original é indicado em itálico e entre parênteses logo após o ano da publicação. Quando o nome específico for considerado sinônimo de outro, o nome atual se encontra indicado, em itálico após o sinal aritmético de igualdade.

Abreviaturas como arm., det., faz., fr., lâm., prot. e s/, foram utilizadas, respectivamente, para as palavras, armadilha, determinado, fazenda, frasco, lâ- mina, protocolo e sem. Quando a preparação do material não foi feita pelo autor da espécie, o preparador é assinalado no texto precedido da abreviatura prep.

Quando os nomes dos colecionadores não estão escritos por extenso é porque constam do rótulo apenas as iniciais.

Dados adicionais, que não constam da etiqueta do exemplar tipo, foram retirados da literatura original, estão indicados entre colchetes.

Dados adicionais que não constam da etiqueta e nem da literatura original e que foram indicados para uma melhor compreensão do texto, estão indicados entre aspas e entre parênteses.

Dados que constam das etiquetas em outro idioma que não o português ou cuja grafia caiu em desuso ou ainda os dados geográficos que sofreram alteração físico-política se encontram entre aspas.

Dados que constam de etiquetas incluídas posteriormente à publicação original estão indicados no texto.

\section{Subfamília CERATOPOGONINAE Newman, 1834 Tribo CERATOPOGONINI Newman, 1834}

Gênero Bahiahelea Wirth, 1992

brasiliensis Wirth, 1992. Parátipos macho e fêmea (Dip.Cer.215, 216) BRASIL, Bahia, Itajuipe, Fazenda Almirante, 9.XII.1988, 23.XII.1988, J. A.Winder, ["emergency trap"].

\section{Gênero Downeshelea Wirth \& Grogan, 1988}

bicornis Felippe-Bauer \& Quintelas, 1993b. Holótipo macho (Dip.Cer.208). BRASIL, Rio de Janeiro, Rio Bonito, Centro, 29.III.1989, Fundação Estadual de Engenharia do Meio Ambiente (FEEMA).

carioca Tavares \& Pereira, 1978 (Monohelea). Parátipos 3 machos e 1 fêmea (Dip.Cer.132-135). ("BRASIL"), Rio de Janeiro, ("Rio de Janeiro"), Tijuca, Gávea Pequena, II.1977, Tavares \& Paiva.

castroi Tavares \& Pereira, 1978 (Monohelea). Parátipo fêmea (Dip.Cer.136). ("BRASIL"), Rio de Janeiro, ("Rio de Janeiro"), Tijuca, Gávea Pequena, II.1977, Tavares \& Paiva.

fluminensis Felippe-Bauer \& Quintelas, 1993a. Holótipo macho (Dip.Cer.202). BRASIL, R.[io] [de] J.[aneiro], A.[rraial] do Cabo, Figueira, R.("ua") São Januário, 29.III.1989, Fundação Estadual de Engenharia do Meio Ambiente (FEEMA). Parátipos: 1 macho (Dip.Cer.203). BRASIL, R.[io] [de] J.[aneiro], Itaboraí, Centro, R.("ua") Dr. Mesquita[s/ n],22.V.1989, FEEMA; 1 macho (Dip.Cer.204). BRASIL, R.[io] [de] J.[aneiro], Casimiro de Abreu, Centro, R.("ua") Padre Anchieta 234, 10.IV.1989, 
FEEMA; I macho (Dip.Cer.205). BRASIL, R.[io] [de] J.[aneiro], S.[ão] Pedro da Aldeia, Estação, R.("ua") Coronel F. Pinheiro 43, 27.III.1989, FEEMA.

quasidentica Felippe-Bauer \& Quintelas, 1993a. Holótipo macho (Dip.Cer.206). BRASIL, Rio de Janeiro, S.[ão] Pedro da Aldeia, Estação, R.("ua") Coronel F. Pinheiro 43, 27.III.1989, Fundação Estadual de Engenharia do Meio Ambiente (FEEMA). Parátipo macho (Dip.Cer.207). Ibidem.

Gênero Monohelea Kieffer, 1917

affinis Felippe-Bauer \& Spinelli, 1991. Holótipo macho (Dip.Cer.199). [BRASIL], Amazonas,("São Paulo de Olivença"), Feijoal, Rio Solimões, X.1978, Shelley \& Luna. Alótipo fêmea (Dip.Cer.200). Ibidem.

aguirrei Tavares \& Souza, $1980=$ maculipennis (Coquillett) partim: parátipos n. Dip.Cer.124-127. Holótipo macho (Dip.Cer.121). ("BRASIL"), Rio de Janeiro, ("Rio de Janeiro"), Jacarepaguá, Tanque, IV.1972, Tavares \& Souza. Parátipos: 2 machos (Dip.Cer.122-123). Ibidem, exceto III.1972; 1 macho e 3 fêmeas (Dip.Cer.124-127). Ibidem, exceto VI,VII,VIII.1972.

archibaldoi Tavares \& Souza, 1980. Holótipo macho (Dip.Cer.128). ("BRASIL"), Rio de Janeiro, ("Rio de Janeiro"), Jacarepaguá, Tanque, VII.1972, Tavares \& Souza. Parátipos: 1 fêmea (Dip.Cer. 131). Ibidem; 2 fêmeas (Dip.Cer.129,130). Ibidem, exceto III, IV.1972.

roraimensis Felippe-Bauer \& Spinelli, 1991. Holótipo macho (Dip.Cer.201). BRASIL, Roraima, Missão Catrimani, I.1979, C.A.E.

maya Felippe-Bauer, Huerta \& Ibañez-Bernal, 2000. Holótipo macho (Dip.Cer.278), MÉXICO, Yucatán, Res(“erva”). Ria Lagartos, Loc(“alidade”) Ojo de Agua, 31.VIII.1995, Navarro C., Paz R. \& Huerta H., "veg("etacíon") Petén, malaise t[rap]" (CONABIO 9703648). Alótipo fêmea (Dip.Cer.279).MÉXICO, Yucatán, Ria Lagartos, "10 km al E. sobre carr ("etera"). San Felipe-Las Coloradas", 04.IV.1997, IBS, MMCL, PMS \& HH, "Petén [veg.("etation”)], malaise t[rap]". Parátipos: 9 machos e 9 fêmeas (Dip.Cer.280-297) mesmos dados do alótipo; 1 machoe 4 fêmeas (Dip.Cer.298-302). MÉXICO, Yucatán, Celestún, Rancho Loma Bonita, 15 km Carr("etera") Celestún-Kinchil, 09.IV.1997, IBS, HJH, MML \& MSP, "Petén [veg.("etacíon")], malaise t[rap]".

\section{Tribo CULICOIDINI Kieffer, 1911}

Gênero Culicoides Latreille, 1809

acotylus Lutz, 1913 (n.2984). Série típica (3181-3182). BRASIL, Mato Grosso, [Salto Augusto], Faz.("enda”) Ojucuí, [Rio Tapajoz], s/data, [Murillo
Campos]. Coleção Costa Lima.

aethionotus Wirth \& Blanton, $1955 \mathrm{~b}=$ wokei Fox. Parátipos (em alfinete): 2 fêmeas (Dip.Cer.336, 337), 1 fêmea sem abdomen e antenas (Dip.Cer.338). PANAMÁ, Zona do Canal, Mojinga Swamp, Fort Sherman, 29.X.1951, 2.I.1952, I.1953, F.S.Blanton, ["light trap"].

almirantei Wirth \& Blanton, 1959(n.5840). Parátipo fêmea (5115). PANAMÁ, Bocas del Toro, Almirante, 2.04 milhas, 15.IV.1953, F.S.Blanton, arm.luz. Coleção Costa lima.

aragaoi Tavares \& Luna Dias, 1980. Holótipo macho (Dip.Cer.1). BRASIL, Rio de Janeiro, ("Rio de Janeiro"), Jacarepaguá, Pau da Fome, II.1974, Tavares \& Ruiz. Parátipos: 5 machos (Dip.Cer.2-6). Ibidem, exceto as datas III e IV.1973, IX.1974, X.1975; 3 fêmeas (Dip.Cer.7-9). Ibidem, exceto II. 1978, Tavares.

archboldoi Wirth \& Blanton, 1970a. Parátipos: 1 macho (Dip.Cer.10). DOMINICA, B[ritish]. W[est].I[ndies]., Clarke Hall, 19.VII.1964, T.J.Spilman,"light trap"; 1 macho (Dip.Cer.11). Ibidem, exceto VI.1964, O.S.Flint; 3 fêmeas (Dip.Cer.12-14). DOMINICA, B[ritish]. W[est]. I[ndies]., Point Casse, VI. e 15-16.VI.1964, O.S.Flint, "light trap". Na literatura original, para a localidade de Clarke Hall, não constam as datas de VI , 1516.VI. e 19.VII.1964, mas sim IV-V e VIII.1964.

bambusicola Lutz, 1913 (n.2982). Série típica: 2 fêmeas (em alfinete, fr. 1259) contendo no rótulo a localidade de Petrópolis e em "taquaraçu", s/data, Costa Lima prep.; 7 lâminas (3171-3177): 1 macho (3171); 3 machos (3173), 2 exemplares s/ antenas e asas e 1 exemplar s/ asas; asa e antenas de macho (3172); asa de macho (3176); cabeça, asa e 3 patas de fêmea (3174); asa de fêmea (3175); abdomen de fêmea (3177), s/ loc., s/ data, Costa Lima prep. em III-1937. Coleção Costa Lima. Aparentemente as lâminas 3174,3175 e 3177 correspondem a 1 exemplar fêmea e as lâminas 3172 e 3176 possuem estruturas de exemplares montados na lâmina 3173 .

barthi Tavares \& Souza, 1978. Parátipos 2 machos e 1 fêmea (Dip.Cer.15-17). BRASIL, Rio de Janeiro, ("Rio de Janeiro"), Jacarepaguá, Pau da Fome, IX.1974, XI.1975, Tavares \& Ruiz.

batesi Wirth \& Blanton, 1973. Parátipos: 6 fêmeas (Dip.Cer.18, 19, 22-25).BRASIL, Pará, Belém, Floresta da Área de Pesquisas Ecológicas do Guamá (APEG), IV, VIII.1969, T.H.G.Aitken, "light trap", "varzea ground"; 2 fêmeas (Dip.Cer.20, 21). Ibidem, exceto os dados VII, IX. 1969, "varzea canopy".

belemensis Wirth \& Blanton, 1973. Parátipos: 6 fêmeas (Dip.Cer.26, 27, 29, 30, 32, 33). BRASIL, Pará, 
Belém, Floresta da Área de Pesquisas Ecológicas do Guamá (APEG), IV, VII, VIII.1969, T.H.G. Aitken, "light trap", "varzea canopy"; 1 fêmea (Dip.Cer.31) Ibidem, exceto os dados VIII.1969,"varzea ground"; 1 fêmea (Dip.Cer.28). Ibidem, exceto os dados XII.1969, "tree canopy".

brasilianum Forattini, 1956 (n.5821). Parátipos (em alfinete): 2 fêmeas (fr.1653) e 2 fêmeas (fr.1654). BRASIL, São Paulo, São Vicente, 15.X.1955, 27.X.1955, X.1955, XI.1955, Forattini e Rabello. Coleção Costa Lima.

bredini Wirth \& Blanton, 1970a. Parátipos 2 machos e 3 fêmeas (Dip.Cer.34-38). DOMINICA, B[ritish]. W[est].I[ndies]., Clarke Hall, 19.VII,VIII, 1-8.VIII, 915.VIII, 10.IX.1964, T.J.Spilman, "light trap".

brownei Spinelli, in Spinelli et al., 1993. Parátipos 10 fêmeas (Dip.Cer.242-251).COLÔMBIA, Valle, Rio Raposo, III, VII, XII.1963 e IV, V, VIII.1965, V.H.Lee, "light trap". Na literatura original não consta a data de IV.1965, mas sim II, III, V, VII e VIII.1965.

cancer Hogue \& Wirth, 1968. Parátipos: 1 macho (Dip.Cer.39). COSTA RICA, [Puntarenas, Golfo de Nicoya], Boca de Barranca, 6.VII.1967, C.L.Hogue e [D.B.Bright], "crab hole" [Cardisoma crassum]; 1 fêmea (Dip.Cer.40) COSTA RICA, [Guanacaste], Península de Nicoya, Playas del Coco, 18.VII.1967, C.L.Hogue e [D.B.Bright], "crab hole" [C.crassum].

carpenteri Wirth \& Blanton, 1953a. Parátipos 2 fêmeas (alfinetes $n^{\text {os }}$ Dip.Cer.303, 304). PANAMÁ, Zona do Canal, Mojinga Swamp, Fort Sherman, 10.XII.1951, F.S.Blanton, "light trap".

carvalhoi Wirth \& Blanton, 1973. Parátipos 2 machos e 5 fêmeas (Dip.Cer.41-47). BRASIL, Pará, Belém, Floresta da Área de Pesquisas Ecológicas do Guamá(APEG), VII,VIII.1970, T.H.G. Aitken, "light trap", em terra firme. Na literatura original não constam as datas de VII, VIII.1970, mas sim IX-XI. 1970.

crescentis Wirth \& Blanton, 1959. Parátipo fêmea ( alfinete $\mathrm{n}^{\mathrm{O}}$ Dip.Cer.339) contendo cabeça e uma pata posterior. PANAMÁ, Bocas del Toro, Almirante, X. 1952, F.S.Blanton, ["light trap"]. Na literatura original não consta a data de X.1952, mas sim XI.1952 a I. 1953.

deanei Felippe-Bauer \& Wirth, 1987. Holótipo fêmea (Dip.Cer.137). BRASIL, Rio de Janeiro, ("Rio de Janeiro"), Jacarepaguá, Represa dos Ciganos, 20.XI.1984, (“M.L.”)Felippe, "biting man”.Alótipo macho (Dip.Cer.138). BRASIL, Rio de Janeiro, ("Rio de Janeiro"), Jacarepaguá, Pau da Fome, IX.1973,Tavares \& Ruiz,"light trap". Parátipos: 5 fêmeas (Dip.Cer.141, 148-151) mesmos dados do holótipo; 6 fêmeas (Dip.Cer.142-147) Ibidem, exceto 25.IX.1984; 1 macho e 1 fêmea (Dip.Cer.139, 140) mesmos dados do alótipo, exceto III, XI.1973.

dicrourus Wirth \& Blanton, 1955b. Parátipos: 2 fêmeas (alfinetes n ${ }^{\circ s}$ Dip.Cer.340, 341). PANAMÁ, Bocas del Toro, Almirante, X.1952, F.S.Blanton, ["light trap"]; 1 macho e 1 fêmea (alfinetes nos Dip.Cer.342, 343) PANAMÁ, Zona do Canal, Mojinga Swamp, Fort Sherman, 29.X.1951, 5.XII.1951, F.S.Blanton, "light trap".

dominicanus Wirth \& Blanton, 1970b. Parátipos 1 macho e 1 fêmea (Dip.Cer.48, 49). DOMINICA, B[ritish]. W[est]. I[ndiens]., Point Casse, 15-16.VI, 23.VI.1964, O.S.Flint, "light trap".

duartei Tavares \& Luna Dias, 1980. Holótipo macho (Dip.Cer.58). BRASIL, Rio de Janeiro, ("Rio de Janeiro"), Jacarepaguá, Pau da Fome, III.1976, O. Tavares. Parátipos: 1 macho (Dip.Cer.59) mesmos dados do holótipo, exceto I.1976, A. Farias; 5 fêmeas (Dip.Cer.60-64) mesmos dados do holótipo, exceto XI.1975, XII.1976 e I.1977, O.Tavares. Na literatura original não consta a data de XI. 1975.

eldridgei Wirth \& Barretto, 1978. Parátipo fêmea (Dip.Cer.65). COLÔMBIA, [Valle], Rio Raposo, IV.1963, V.H.Lee, "light trap".

espinolai Felippe-Bauer \& Lourenço-de-Oliveira, 1987. Holótipo fêmea (Dip.Cer.157). BRASIL, Minas Gerais, Felixlândia, Faz. Exp. Guimarães Rosa, Empresa de Pesquisa Agropecuária de Minas Gerais (EPAMIG), 28.X.1986, R.L.[ourenço] de Oliveira, "biting man". Parátipos: 5 fêmeas (Dip.Cer.158162) mesmos dados do holótipo, exceto Vargem Grande, Manga Velha, 21.X.1986, "biting on marmoset"; 2 fêmeas (Dip.Cer.163, 164) mesmos dados do holótipo, exceto Ponte Nova, 01, 02.XI.1986; 2 fêmeas (Dip.Cer.165, 166) Ibidem, exceto Limeira, 03.XI.1986.

evansi Wirth \& Blanton, 1959. Parátipos: 1 macho (Dip.Cer. 371), 1 fêmea (alfinete $\mathrm{n}^{\circ}$ Dip.Cer.344). PANAMÁ, Chiriqui, [El] Volcan, 23.III, 13.IV.1954, F.S.Blanton, ["light trap"]. Na literatura original não consta a data de 23.III mas sim 25.III.

fernandoi Tavares \& Souza, 1979. Parátipos 2 fêmeas (Dip.Cer.66, 67). BRASIL, Rio de Janeiro, Sumidouro, Sítio Peri-Ceci, III.1978, Tavares \& Souza.

fieldi Wirth \& Blanton, 1956a (n.5857). Parátipo macho (5135). PANAMÁ, Zona do Canal, Huile Sia Clara, 13.X.!952, F.S.Blanton,'light trap". Coleção Costa Lima.

flavivenulus Costa Lima, 1937a (n.3008). Cótipos 2 fêmeas (3221). BRASIL, Bahia, s/ data e col.. Coleção Costa Lima.

franklini Spinelli, in Spinelli et al., 1993. Parátipos: 1 macho e 2 fêmeas (Dip.Cer.237-239). COLÔMBIA, [Choco], Teresita, 5, 30.VII.1967, [D.G.Young]; 1 fê- 
mea (Dip.Cer.240) PANAMÁ, Zona do Canal, Barro Colorado Island, VII.1967, W.W.Wirth, "light trap"; 1 fêmea (Dip.Cer.241) PANAMÁ, Zona do Canal, Summit Gardens, VII.1967, F.S.Blanton, "light trap".

fusipalpis Wirth \& Blanton, 1973. Parátipos: 2 fêmeas (Dip.Cer.51, 53). BRASIL, Pará, Belém, Floresta da Área de Pesquisas Ecológicas do Guamá (APEG), V.1969, T.H.G. Aitken, "light trap", terra firme; 5 fêmeas (Dip.Cer.52, 54-57) Ibidem, exceto VI.1969.

galindoi Wirth \& Blanton, 1953a. Parátipo fêmea (alfinete ${ }^{\circ}$ Dip.Cer.345). PANAMÁ, [Zona do Canal], Mojinga Swamp, [Fort Sherman], 15.VIII.1951, F.S.Blanton, "light trap".

glabelus Wirth \& Blanton, 1956a. Parátipos: 2 fêmeas (Dip.Cer.369, 370), 1 macho (lâm.5147 com prot. $n^{\circ}$ 5867, Coleção Costa Lima). PANAMÁ, Zona do Canal, Mojinga-Swamp, 18.XII.1951, 13.X.1952, F.S.Blanton, "light trap".

goeldii Wirth \& Blanton, 1973. Parátipos 1 macho e 1 fêmea (Dip.Cer.68, 69). BRASIL, Pará, Belém, Floresta da Área de Pesquisas Ecológicas do Guamá (APEG), 8.X.1970, I.1970, T.H.G. Aitken, "light trap", terra firme.

guamai Wirth \& Blanton, 1973. Parátipos 2 machos e 2 fêmeas (Dip.Cer.70-73). BRASIL, Pará, Belém, Floresta da Área de Pesquisas Ecológicas do Guamá (APEG), XII.1969, II, 17.IX., 6.X.1970, T.H.G.Aitken, "light trap", terra firme.

guttatus Coquillett, 1904 (Ceratopogon) (n.2407). Lectótipo fêmea (2820). BRASIL, São Paulo, ("São Paulo"), Serra da Cantareira, s/data, [A.Lutz]. Costa Lima prep. em V.1937. Coleção Costa Lima. (Lectótipo designado por Fox, 1958).Contém rótulo "de um exemplar procedente dos E.("stados") Unidos".

horticola Lutz, 1913 (n.2405). Série típica composta de 4 lâminas: asa danificada de fêmea (2815); 1 fêmea sem asas, antenas e genitália (2816); asa de fêmea (3242); asa de fêmea (3243). BRASIL, São Paulo, Tatuhy, s/data e col., Costa Lima prep. em V.1937 (3242-3243); ibidem [Tatuhy], Costa lima prep.VI.1936 (2815-2816). Coleção Costa Lima . Aparentemente as lâminas 2815 e 2816 pertencem a 1 exemplar fêmea.

insignis Lutz, 1913 (n.5827). Lectótipo macho (5092), s/cabeça e asas. BRASIL, s/ localidade, data e col.. Coleção Costa Lima. O Lectótipo macho está montado na lâmina 5092 juntamente com 1 fêmea s/asas, indicada como "lectoparátipo". (Lectótipo designado por Wirth \& Blanton, 1956c).

kampa Felippe-Bauer, Veras \& Castellon, in Felippe-Bauer et al., 2000. Parátipos 2 fêmeas
(Dip.Cer.276, 277). BRASIL, Acre, Juazeiro, Rio Azul, 24, 25.XI.1996, E.F. Morato, "light trap".

lenti Tavares \& Luna Dias, 1980. Holótipo macho (Dip.Cer.74). BRASIL, Rio de Janeiro, ("Rio de Janeiro"), Tijuca, Gávea Pequena, Fundação Estadual de Engenharia do Meio Ambiente (FEEMA), XI.1974. Parátipos 3 machos (Dip.Cer.75-77) Ibidem, exceto VIII, XI.1974.

lobatoi Felippe-Bauer \& Quintelas, 1994. Holótipo fêmea (Dip.Cer.209). BRASIL, Rio de Janeiro, Nova Friburgo, Furnas do Catete, X-XI.1987, FelippeBauer, "light trap". Parátipos: 4 fêmeas (Dip. Cer.210213) Ibidem, exceto 4-13.X.1987, 4.XII.1987 11.I.1988, 11.III-14.IV.1988; 1 fêmea (Dip.Cer.214) BRASIL, Rio de Janeiro, ("Rio de Janeiro"), Jacarepaguá, Pau da Fome, VII.1974, Tavares.

lutzi Costa Lima, 1937a (n.2408). Cótipos: 2 fêmeas (em alfinete $\mathrm{n}^{\circ} 2408$ ); 11 lâminas $(2821,3222-3228$, 3230-3232): asa de fêmea (2821, 3223, 3224 e 3232); asas de fêmea (3222); tórax, abdomen e asas de fêmea (3225); tórax e abdomen de fêmea (3226); cabeça e tórax de fêmea (3227); cabeça , tórax e abdomen de fêmea (3228); cabeça de fêmea (3230); toráx e abdomen (3231). BRASIL, Pará, Abaeté, 8.VI.1936, Dr.Evandro Chagas. Coleção Costa Lima. Aparentemente as lâminas 3230 a 3232 pertencem a 1 exemplar fêmea.

macieli Tavares \& Ruiz, 1980. Parátipos 1 macho e 3 fêmeas (Dip.Cer.117-120). [BRASIL], Rio de Janeiro, ("Rio de Janeiro"), Jacarepaguá, Pau da Fome, IV, V.1973, Tavares \& Ruiz. Na literatura original não consta como parátipos 1 macho e 3 fêmeas, e sim, 4 machos e 2 fêmeas.

marinkellei Wirth \& Lee, 1967. Parátipos 2 fêmeas (Dip.Cer.78, 79). COLÔMBIA. Cauca, Paramo de Puracé, Laço Buey, 28.X.1964. V.H.Lee, "biting man", $3250 \mathrm{~m}$.

marshi Wirth \& Blanton, 1956b. Parátipo fêmea (alfinete $n^{\circ}$ Dip.Cer.346). PANAMÁ, Bocas del Toro, Almirante, III. 1953, F.S.Blanton ["light trap"].

maruim Lutz, 1913 (n.5802 e 2985). Série típica: 1 macho (em alfinete, fr. 1650); 4 lâminas (5085; 31833185): pupa de macho (5085), 9 fêmeas e 5 machos (3183), asa de fêmea $(3184,3185)$; 20 fêmeas (em álcool, fr. 1260). BRASIL, Rio de Janeiro, ("Rio de Janeiro"), Manguinhos, 25-VI-1913 (indicada apenas no frasco 1650 e na lâmina 5085), s/ col., em mangue, Forattini e Galvão prep. em IV.1955 (fr.1650, lam.5085), Costa Lima prep. em II.1937 (3184, 3185). Coleção Costa Lima. Aparentemente a lâmina 5085 e o frasco 1650 pertencem a 1 exemplar macho.

metagonatus Wirth \& Blanton, 1956b. Parátipos: 1 fêmea (Dip.Cer.377). PANAMÁ, [Coclé], El Valle, 
25.VI.1954, (“U.S.Army”) 25 Med. Det., "light trap"; 1 macho e 1 fêmea (lâm. 5164-5165 com prot. n ${ }^{\circ}$ 5881, Coleção Costa Lima), Ibidem, exceto [F.S.Blanton]; 3 fêmeas (alfinetes $n^{\circ}$ Dip.Cer. 347, 348; lâm. $n^{\circ}$ Dip. Cer. 376). PANAMÁ, Panamá, Cerro Campana, 17, 18.VIII.1954 "U.S.Army 25 Med Det, light trap"; 1 macho (Dip.Cer. 375). PANAMÁ, [Bocas del Toro], Almirante, XI. 1952, F.S.Blanton. Na literatura original, para a localidade de El Valle, não consta a data de 25.VI.1954, mas sim II.1953 e, para a localidade de Cerro Campana, não consta a data de 17, 18.VIII.1954, mas sim 3.VII., 19.IX.1951, 24.I e 24.IV.1952.

minasensis Felippe-Bauer, 1987. Holótipo fêmea (Dip.Cer.152). BRASIL, Minas Gerais, Felixlândia, Ponte Nova, 30.X.1986, R.L.[ourenço] de Oliveira, "biting on marmoset". Parátipos: 1 fêmea (Dip.Cer.155) Ibidem; 1 fêmea (Dip.Cer.154) Ibidem, exceto "biting man"; 1 fêmea (Dip.Cer.156) BRASIL, Minas Gerais, Felixlândia, Limeira, 3.XI.1986, R.L.[ourenço] de Oliveira, "biting man"; 1 fêmea (Dip.Cer.153) BRASIL, Minas Gerais, Felixlândia, Faz. Exp. Guimarães Rosa, Empresa de Pesquisa Agropecuária de Minas Gerais (EPAMIG), 31.X.1986, R.L.[ourenço] de Oliveira, "animal trap".

neoparaensis Tavares \& Souza, 1978. Alótipo fêmea (Dip.Cer.80). ("BRASIL"), Rio de Janeiro, ("Rio de Janeiro”), Jacarepaguá, X.1975, Tavares \& Ruiz. Parátipos 2 machos e 3 fêmeas (Dip.Cer.81-85) Ibidem, exceto, III. 1974, IX, X, XI.1975. Na literatura original não consta o alótipo.

nigrigenus Wirth \& Blanton, 1956b. Parátipo fêmea (alfinete $\mathrm{n}^{\circ}$ Dip.Cer.305). PANAMÁ, Bocas del Toro, Almirante, I.1953, F.S.Blanton, ["light trap"].

paraensis Goeldi, 1905 (Haematomyidium). Neótipo fêmea (Dip.Cer.197). BRASIL, ("Pará"), Belém, Empresa Brasileira de Pesquisa Agropecuária (EMBRAPA), 11.XI.1986, A.L. Hoch, "biting man 18:00 h". (Neótipo designado por Wirth \& FelippeBauer, 1989).

paraignacioi Spinelli, in Spinelli et al., 1993. Parátipos: 4 fêmeas (Dip.Cer.227-230). COLÔMBIA, [Choco], Curiche, 12.XI.1967, [D.G.Young], "light trap"; 1 fêmea (Dip.Cer.231). COLÔMBIA, Antioquia, "near rio Anori, tropic rain forest", IX.1970, D.G.Young, "blacklight trap"; 3 fêmeas (Dip.Cer.232-234). BRASIL, Pará, Belém, Floresta da Ârea de Pesquisas Ecológicas do Guamá (APEG), I,V,VII.1969, T.H.G.Aitken, "light trap", terra firme; 2 fêmeas (Dip.Cer.235, 236). COSTA RICA, Puntarenas, Villa Neilly, 21.VI.1964, REW. Na literatura original, para a localidade de Curiche, não consta a data de 12.XI.1967, mas sim 12.VI.1967. patulipalpis Wirth \& Blanton, $1959=$ rangeli Ortiz \& Mirza. Parátipos: 4 fêmeas (alfinetes ${ }^{\circ}$ s Dip.Cer.349-351, lâm. nº Dip.Cer. 367). PANAMÁ, Bocas del Toro, Almirante, X e XI.1952, III. 1953, F.S.Blanton, ["light trap"]; 1 fêmea (Dip.Cer. 368).[PANAMÁ], Zona do Canal, Mojinga Swamp, XI.1951, F.S.Blanton, "light trap". Na literatura original não consta a data de XI.1951 mas sim 24.X e 10.XII.1951.

phaeonotus Wirth \& Blanton, 1959. Parátipos: 1 macho e 1 fêmea $\left(5175,5176\right.$, com prot. $\mathrm{n}^{\circ} 5891$, Coleção Costa Lima); 1 fêmea (alfinete ${ }^{\circ}$ Dip.Cer.352). PANAMÁ, Bocas del Toro, Almirante, XI, XI-XII.1952, F.S.Blanton,"light trap".

popayanensis Wirth \& Lee, 1967. Parátipos 2 fêmeas (Dip.Cer.86, 87). COLÔMBIA, Cauca, Paramo de Puracé, 28.4 km leste de Puracé, 18.II.1965, V.H.Lee, "[at] light", $3100 \mathrm{~m}$.

pseudocrescentis Tavares \& Luna Dias, 1980. Holótipo macho (Dip.Cer.116). BRASIL, Rio de Janeiro, ("Rio de Janeiro"), [Tijuca], Gávea [Pequena], VI.1975, Fundação Estadual de Engenharia e Meio Ambiente (FEEMA). Na literatura original não consta a data de VI.1975, mas sim VII.1975.

pusilloides Wirth \& Blanton, 1955a. Parátipos: 2 fêmeas (alfinetes $n^{\circ s}$ Dip.Cer.353, 354), 1 fêmea sem asa, abdomen e antenas (alfinete $\mathrm{n}^{\circ}$ Dip.Cer.355), 1 fêmea sem abdomen e cabeça (alfinete ${ }^{\circ}$ Dip.Cer.356), 1 macho (Dip.Cer. 374). PANAMÁ, Bocas del Toro, Almirante, X. ( Dip.Cer.353, 354), XI. 1952 (Dip.Cer.355, 356, 374), F.S. Blanton, ["light trap"].

rachoui Tavares \& Souza, 1978. Alótipo fêmea (Dip.Cer.88).("BRASIL"), Rio de Janeiro, ("Rio de Janeiro"), Jacarepaguá, Pau da Fome, VI.1976, O. Tavares. Parátipos: 4 machos (Dip.Cer.89-92). Ibidem, exceto XI.1974, XI, XII.1975; 1 fêmea (Dip.Cer.93) mesmos dados do alótipo, exceto XI.1975. Na literatura original não consta o alótipo e a data de XII.1975.

raposoensis Wirth \& Barreto, 1978. Parátipos 1 macho e 2 fêmeas (Dip.Cer.94-96). COLÔMBIA, [Valle], Rio Raposo, IV.1963, 01.VII, VII.1964, V.H.Lee, "light trap".

reticulatus Lutz, 1913 (n.2983). Série típica 7 fêmeas e 1 macho (3180). BRASIL, Bahia, s/data e col.. Coleção Costa Lima.

saintjusti Tavares \& Ruiz, 1980 = ignacioi Forattini. Alótipo fêmea (Dip.Cer.97). [BRASIL], Rio de Janeiro, ("Rio de Janeiro"), Jacarepaguá, Pau da Fome, III.1973, [O.] Tavares \& [R.A.] Ruiz. Parátipos 2 machos e 1 fêmea (Dip.Cer.98-100). Ibidem, exceto IV, V.1973. 
sanmartini Wirth \& Barreto, $1978=$ batesi Wirth \& Blanton. Parátipos 2 machos e 2 fêmeas (Dip.Cer.101-104). COLÔMBIA, [Valle], Rio Raposo, XII.1963, II, III.1964, V.H.Lee, "light trap". Na literatura original não consta a data de XII.1963.

setifer Lutz, 1913 (Centrorhynchus) = caridei Brèthes. Série típica: 14 fêmeas (alfinete ${ }^{\circ}$ Dip.Cer.306); 67 fêmeas ( em álcool, fr.n ${ }^{\circ}$ Dip.Cer. 307 ). [BRASIL], Rio Grande do Sul, Santa Vitória do Palmar,[1912], E.V. Bassewitz. Contém rótulo “ Culicoides caridei Brèthes, det. John Lane, 1944”.

spurius Wirth \& Blanton, 1959 (n.5901). Parátipo macho (5187). PANAMÁ, Zona do Canal, Forte Davis, 7.X.1953, F.S.Blanton,"light trap". Coleção Costa Lima. Na literatura original os dados relacionados com este exemplar constam como sendo de um alótipo.

tavaresi Felippe-Bauer \& Wirth, 1988. Holótipo fêmea (Dip.Cer.167). BRASIL, Rio de Janeiro, Itaguaí, Fazenda da Barrinha, 17.X.1974, Aragão \& Tavares, isca luminosa. Alótipo macho (Dip.Cer.168) Ibidem. Parátipos 12 machos e 14 fêmeas (Dip.Cer.169-194) Ibidem.

tenuilobus Wirth \& Blanton, 1959. Parátipo fêmea (alfinete $\mathrm{n}^{\circ}$ Dip.Cer.357). PANAMÁ, Bocas del Toro, Almirante, 13.X.1952, F.S.Blanton, ["light trap"].

teretipalpis Wirth \& Barreto, 1978. Parátipo fêmea (Dip.Cer.105). COLÔMBIA, [Valle], Rio Raposo, IV.1964, V.H.Lee, "light trap".

tetrathyris Wirth \& Blanton, 1959. Parátipos: 1 fêmea (alfinete $\mathrm{n}^{\circ}$ Dip.Cer.365). PANAMÁ, Zona do Canal, Mojinga Swamp, Fort Sherman, I. 1952, F.S.Blanton, "light trap"; 2 fêmeas (alfinete ${ }^{\circ}$ Dip.Cer. 366; lâm. 5190 com prot. n 5904, Coleção Costa Lima). PANAMÁ, Bocas del Toro, Almirante, X, 2.XII.1952, F.S.Blanton, "light trap"; 1 macho (Dip.Cer. 372). HONDURAS, Lancetilla, 9 st. C.O., s/ data, F.S. Blanton, "light trap"; 1 fêmea (Dip.Cer. 373) Ibidem, exceto 14.I.1954. Na literatura original para a localidade de Mojinga Swamp não consta a data de I. 1952 mas sim I. 1951 e, para a localidade de Lancetilla, não consta F.S. Blanton mas sim P. Galindo.

tidwelli Spinelli, in Spinelli et al., 1993. Parátipos: 1 macho e 5 fêmeas (Dip.Cer.217-222). COSTA RICA, Cartago, Navarro, VI-VIII. 1962, F.S.Blanton, "light trap"; I machoe 2 fêmeas (Dip.Cer.223-225). COLÔMBIA, [Valle], Rio Raposo, IV.1963, III, VI.1964, V.H. Lee, "light trap"; 1 macho (Dip.Cer.226). PANAMÁ, Chiriqui, Fortuna, 11.II.1976, T.H.G. Aitken, "light trap", $1000 \mathrm{~m}$. Na literatura original para a localidade de Rio Raposo, não consta a data de VI.1964. trapidoi Wirth \& Barreto, 1978. Parátipos 1 macho e 2 fêmeas (Dip.Cer.106-108). COLÔMBIA, [Valle], Rio Raposo, I, 28.VII, VIII.1964, V.H.Lee, "light trap". uniradialis Wirth \& Blanton, 1953a. Parátipo fêmea (alfinete $n^{\circ}$ Dip.Cer.358). PANAMÁ, Zona do Canal, Mojinga Swamp, Fort Sherman, 24.X.1951, F.S.Blanton, "light trap".

vernoni Wirth \& Blanton, 1973. Parátipos: 1 macho e 3 fêmeas (Dip.Cer.109-111, 114). COLÔMBIA, [Valle], Rio Raposo, 15.IV, 3-4.VI, X.1964, V.H.Lee, "light trap"; 1 fêmea (Dip.Cer.112). BRASIL, Pará, Belém, Floresta da Área de Pesquisas Ecológicas do Guamá (APEG), X.1969, T.H.G. Aitken, "light trap, varzea canopy"; 1 fêmea (Dip.Cer.113). Ibidem, exceto I.1970, "ground level".

youngi Wirth \& Barreto, 1978. Parátipo 1 fêmea (Dip.Cer.115). COLÔMBIA, Valle, Rio Raposo, II. 1965, V.H.Lee, "light trap".

willistoni Wirth \& Blanton, 1953b. Parátipo fêmea (alfinete ${ }^{\circ}$ Dip.Cer.359). PANAMÁ, Cocle, Rio Hato, 15.I.1952, F.S.Blanton, "light trap".

\section{Tribo PALPOMYIINI Enderlein, 1936}

Gênero Palpomyia Meigen, 1818

guarani Lane, 1946. Parátipos ( em alfinete): 2 fêmeas juntamente com a exúvia da pupa (Dip.Cer.360, 361); 1 fêmea, sem antenas, juntamente com a exúvia da pupa (Dip.Cer.362). BRASIL, Espírito Santo, São João, 6.V.1940,[L. Whitman], em bambu ("Bamboo"); 1 fêmea sem antenas e asas (Dip.Cer.363). BRASIL, [Rio de Janeiro] "D.(istrito) Federal", ("Rio de Janeiro"), Silvestre, 2.II.1940, [L.] Whitman, em "tree hole"; 1 fêmea sob. o n ${ }^{\circ}$ 1276, com a exuvia da pupa em lamínula presa ao próprio alfinete (Dip.Cer.364) . BRASIL, São Paulo, ("São Paulo"), Horto Florestal, V.1944, J. Lamosa. Os dados do exemplar de n 364 não constam da literatura original.

\section{Tribo SPHAEROMIINI Newman, 1834}

Gênero Mallochohelea Wirth, 1962

limitrofe Spinelli \& Felippe-Bauer, 1990a. Parátipo fêmea (Dip.Cer.195). URUGUAI, Artigas, Colonia San Gregorio ("arrocera de Conti"), 8-9.XII.1987, G.[R.] Spinelli, [“CDC trap”].

Gênero Neobezzia Wirth \& Ratanaworabhan, 1972 wirthi Spinelli \& Felippe-Bauer, 1990a. Parátipo fêmea (Dip.Cer.196). ARGENTINA, Corrientes, Departamento de Paniagua, Monte Caseros, 22.X.1987, G.[R.] Spinelli, “a la luz".

Gênero Sphaerohelea Spinelli \& Felippe-Bauer, 1990

biestroi Spinelli \& Felippe-Bauer, 1990b. Parátipo fêmea (Dip.Cer.198). ARGENTINA, Misiones, Posadas, 10.XI.1970, C. Porter \& L. Stange. 


\section{Subfamília FORCIPOMYIINAE Lenz, 1934}

Gênero Forcipomyia Meigen, 1818

\section{Subgênero Forcipomyia Meigen, 1818}

squamitibia Lutz, 1914 (n.3287). Série típica composta de 4 lâminas (3685-3688): 1 fêmea(3685), cabeça de fêmea (3686), 1 fêmea s/ cabeça(3687), 1 fêmea s/ asas, patas e antenas (3688).[BRASIL], ("Rio de Janeiro, Rio de Janeiro"), Manguinhos, 1911 (indicado apenas nos exemplares 3685, 3688), s/ col., arm. luz, Costa Lima prep. em X.1937 (indicado apenas nos exemplares 3686-3688). Coleção Costa Lima. Aparentemente as lâminas 3686 e 3687 correspondem a 1 exemplar fêmea.

Subgênero Lasiohelea Kieffer, 1921

stylifer Lutz, 1913 (Centrorhynchus). Série típica: 3 fêmeas (alfinetes $n^{\circ s}$ Dip.Cer.308, 309). [BRASIL], Minas Gerais, Lassance, s/data e col.[em orelha e cabeça de cavalo]; 14 fêmeas (alfinetes $\mathrm{n}^{\circ \mathrm{s}}$ Dip.Cer.310-323). [BRASIL], Bahia, Villa Nova, 1912, s/ col. [em orelha de cavalo]; 5 fêmeas (lâm. 3698 e 3700, com prot. $n^{\circ} 3293$ - Coleção Costa Lima): 2 fêmeas, 1 fêmea s/cabeça, 1 fêmea s/ patas e c/ antena quebrada, 1 fêmea s/antena, pata posterior e mediana (3698); cabeça de fêmea s/antenas (3700). [BRASIL], ("Bahia"), Villa Nova, s/ data e col., [em orelha de cavalo], C.("osta") L.("ima prep. em") VIII.1937; 7 fêmeas (alfinetes n ${ }^{\circ s}$ Dip.Cer.324-330). [BRASIL], São Paulo, Tatuhy, 1903 (indicado apenas nos exemplares n ${ }^{\circ \mathrm{s}}$ Dip.Cer.324-328), s/col.; 2 fêmeas (lâm. 3699, com prot. $n^{\circ} 3293$-Coleção Costa Lima): 1 fêmea s/ cabeça; 1 fêmea s/ cabeça, patas anteriores, medianas e pata anterior. [BRASIL, São Paulo], Tatuhy, s/ data e col., C.("osta") L.("ima prep. em") VIII.1937. O exemplar n ${ }^{\circ}$ Dip.Cer.329 contém rótulo "holótipo" designado por Lane, 1945. Todos os exemplares não pertencentes a Coleção Costa Lima contém rótulo constando o gênero "Cotocripus" e não Centrorhynchus como consta de literatura original.

\section{Subgênero Lepidohelea Kieffer, 1917}

bahiensis Wirth \& Spinelli, 1992. Parátipos: 1 macho (Dip.Cer.254). BRASIL, Bahia, Itajuípe, Fazenda Almirante, 31.I.1990, J.A.Winder; 1 fêmea (Dip.Cer.255) TRINIDAD (“E TOBAGO”), San Salvador, Santo Augustino, 24.VII.1990, A.M. Young, "reared ex rotting cocoa pod husk".

bicolor Lutz, 1914 (n.3288). Lectótipo macho(3689corpo, 3721-genitália). [BRASIL], ("Rio de Janeiro, Rio de Janeiro"), Manguinhos, s/data e col., arm. luz., Costa Lima prep.. Coleção Costa Lima. (Lectótipo designado por Wirth, 1991).

euthystyla Wirth \& Spinelli, 1992. Parátipos 2 fêmeas (Dip.Cer.256, 257). COLÔMBIA, ("Valle"), Rio Raposo, 23.III.1964, V.H. Lee, "light trap". gravesi Wirth \& Spinelli, 1992. Parátipos: 1 fêmea e 1 macho (Dip.Cer.258, 259). ("E.U.A.”), Carolina do Norte, Highlands, VII-1965, P.M. Marsh, "malaise trap"; 3 fêmeas (Dip.Cer.260-262). ("E.U.A.”), Virgínia do Oeste, Lost River St. Park, Hardy Co("unt") 8-14.VII.1963, K.V. Krombeim, "tent trap".

herediae Wirth \& Spinelli, 1992. Parátipos: 1 fêmea (Dip.Cer.263). [COSTA RICA], Heredia, La Virgem, Finca la Tigra, 14.XI.1981, ${ }^{\mathrm{a} M}$. Young, "[ex] moss in forest"; 1 pupa e 1 larva (Dip.Cer.264, 265). [COSTA RICA, Limon]. Squirres, Hacienda Theobroma, 1.VI.1956, L.G. Saunders, "[ex] coconut husks".

hobbsi Wirth \& Spinelli, 1992. Parátipos: 4 machos (Dip.Cer.266-269). DOMINICA, B.("ristish") W.("est") I.("ndies"), Pont Casse, "1.6mi. W.", VI.1964 (266-267) e 15-16.VI.1964 (268, 269). O.L.Flint, "light trap"; 3 fêmeas (Dip.Cer.270-272). DOMINICA, B.("ritish") W.("est") I.("ndies"), Clark Hall, 1-8.VIII.1964 (271, 272) e 15.IX.1964 (270), T.S.Spilman, "light trap"; 1 fêmea (Dip.Cer.273). DOMINICA, B.("ristish") W.("est") I.("ndiens"), Mouth Layou R[iver], 20.I.1965, W.W. Wirth, "light trap".

weemsi Wirth \& Spinelli, 1992. Parátipos: 1 macho (Dip.Cer.274). ("E.U.A.”), Florida, Highlands Co("unt"), Lake Placid, Archbold Biological Station, 12.IX.1989, W.W. Wirth, "malaise trap"; 1 fêmea (Dip.Cer.275). ("E.U.A.”), Florida, Alachua Co("unt"), Gainesville, Chantilly Acres, VI.1967, F.S. Blanton, "light trap".

\section{Subgênero Microhelea Kieffer, 1917}

obesa Costa Lima, 1928. Holótipo fêmea em 3 lâminas: corpo (500); antena, asa e palpo $(501,502)$. BRASIL, (“Amazonas"), São Gabriel ("da Cachoeira”), Alto do Rio Negro, 11.X.1927, J.F.Zikán, em fasmídeo. Coleção Costa Lima.

squamosa Lutz, 1914 (n.3281). Série típica composta de 13 lâminas: 3 machos e 3 fêmeas (3650), sendo 1 fêmea sem cabeça; 2 fêmeas (3651), sendo uma sem asas e pata posterior e outra sem cabeça; cabeça de fêmea (3652); patas de fêmea (3653); 1 macho (3654); 1 macho sem cabeça e genitália (3655); genitália de macho (3656); cabeça de macho (3657); 3 machos e 3 fêmeas (3658); 1 fêmea sem asa (3659); asa de fêmea (3660); asas de macho (3661); asa de fêmea (3662). [BRASIL], ("Rio de Janeiro, Rio de Janeiro"), Manguinhos, 1911 (indicado apenas nas lâminas 3653, 3655-3657, 3662), [Lutz , arm. luz], Costa Lima prep. VIII.1937(3650-3654,3658-3662)eIX.1937(36553657). Coleção Costa Lima. A lâmina 3650 e 3658 contém o seguinte dado "num vidro com rótulo Forcipomyia maculipes". As lâminas 3651, 3652, 3655-3657, 3660-3662 contém o seguinte dado "material típico". Aparentemente a lâmina 3652 contém a 
cabeça do exemplar fêmea da lâmina 3651, as lâminas 3655-3657 pertencem a 1 exemplar macho e as lâminas 3659 e 3660 pertencem a 1 exemplar fêmea. $\mathrm{Na}$ literatura original consta apenas 2 machos como da série típica, os quais são base da descrição original.

\section{Subgênero Phytohelea Remm, 1971}

bromelicola Lutz, 1914 (Ceratopogon). Série típica 2 machos (alfinete $\mathrm{n}^{\circ}$ Dip.Cer.331), com genitália de 1 dos exemplares em lamínula presa ao próprio alfinete.[BRASIL], Rio de Janeiro, ("Rio de Janeiro"), Ilha do Governador, s/data e col. Contém rótulo "holótipo, designado por Lane, 1945".

Subgênero Pterobosca Macfie, 1932

macfiei Costa Lima, 1937b (Pterobosca) $=$ fusicornis (Coquillett). Holótipo fêmea (n.3289) em 3 lâminas: asa (1075), cabeça (1552), fêmea s/ asas e cabeça (1553). ("BRASIL"). [Rio de Janeiro], "Distrito Federal", ("Rio de Janeiro"), Tijuca, Alto da Boa Vista, 13-IV-1930, Carlos Alberto C.("ampos") Seabra, em [asas] de Odonata, prep. em IV.1930, det. em IX.1937. Parátipo fêmea (n.3290) em 2 lâminas: fêmea s/ asas (1085), asas (3692). ("BRASIL"). [Rio de Janeiro], ("Rio de Janeiro"), Tijuca, Alto da Boa Vista, 20.IV.1930, Carlos Alberto ("Campos") [Seabra], em [asas de] Odonata, Anisoptera, ("det. em") VIII.1937. Coleção Costa Lima.

\section{Subfamília DASYHELEINAE Lenz, 1934}

\section{Gênero Dasyhelea Kieffer, 1911}

pusilla Lutz, 1913 (Centrorhynchus). Série típica (em alfinete): 1 fêmea (Dip.Cer.332) com rótulo "holótipo"designado por Lane, 1945 e contendo pata mediana em lamínula presa ao próprio alfinete; 1 fêmea (Dip.Cer.333) com asa em lamínula presa ao próprio alfinete. [BRASIL], Rio de Janeiro, [Serra da Bocaina, Fazenda] Bonito, I.1912, s/col. Contém rótulo "John Lane det. 1944".

filibranchia Lutz,1914 (Ceratopogon). Série típica 1 fêmea s/ cabeça (alfinete $n^{\circ}$ Dip.Cer.334). [BRASIL, Rio de Janeiro], ("Rio de Janeiro"), [Manguinhos], VI.1913, [A. Lutz, arm. luz]. Contém rótulo "criado de ninfas achadas no mangue".

\section{Subfamília LEPTOCONOPINAE Noè, 1907}

Gênero Leptoconops Skuse, 1889

\section{Subgênero Leptoconops Skuse, 1889}

brasiliensis Lutz, 1913 (Tersesthes). Série típica 3 fêmeas ( alfinete ${ }^{\circ}$ Dip.Cer.335 ). BRASIL, [Pará], Rio Tocantins, 1912, A("strogildo") Machado.

\section{REFERÊNCIAS}

Cardozo-de-Almeida M, Linardi PM, Costa J 1999. The type specimens of sucking lice (Anoplura) deposited in the Entomological Collection of Instituto
Oswaldo Cruz, Rio de Janeiro, RJ, Brazil. Mem Inst Oswaldo Cruz 94: 625-628.

Cesar Pinto 1930. Arthropodes Parasitos e Transmissores de Doenças, Tomo II, Editora Pimenta de Mello e Companhia, Rio de Janeiro, p. 396-846.

Coquillett DW 1904. A new Ceratopogon from Brazil. JNY Entomol Soc 12: 35-36.

Costa Lima A da 1928. Ceratopogonineos ectoparasitos de phasmideos. Mem Inst Oswaldo Cruz 3 (Suppl.): 84-85.

Costa Lima A da 1937a. Chave das espécies de Culicoides da região neotrópica (Diptera: Ceratopogonidae). Mem Inst Oswaldo Cruz 32: 411-422.

Costa Lima A da 1937b. Primeira espécie americana do gênero Pterobosca (Diptera: Ceratopogonidae). Mem Inst Oswaldo Cruz 32: 615-616.

Felippe-Bauer ML 1987. A new Neotropical Culicoides from Minas Gerais (Diptera: Ceratopogonidae). Mem Inst Oswaldo Cruz 82: 147-148.

Felippe-Bauer ML, Quintelas AR 1993a. Two new Brazilian predaceous midges of the genus Downeshelea Wirth and Grogan (Diptera: Ceratopogonidae). Mem Inst Oswaldo Cruz 88: 33-38.

Felippe-Bauer ML, Quintelas AR 1993b. Downeshelea bicornis, a new neotropical predaceous midge from Brazil (Diptera: Ceratopogonidae). Mem Inst Oswaldo Cruz 88: 185-187.

Felippe-Bauer ML, Quintelas AR 1994. Culicoides lobatoi, a new Brazilian biting midge of the Limai group (Diptera: Ceratopogonidae). Mem Inst Oswaldo Cruz 89: 25-27.

Felippe-Bauer ML, Lourenço-de-Oliveira R 1987. A new neotropical biting midge of the Culicoides debilipalpis group (Diptera: Ceratopogonidae). Mem Inst Oswaldo Cruz 82: 149-150.

Felippe-Bauer ML, Spinelli GR 1991. New Brazilian predaceous midges of the genus Monohelea Kieffer, and redescription of $M$. guaimiesi (Diptera: Ceratopogonidae). Mem Inst Oswaldo Cruz 86: 201-207.

Felippe-Bauer ML, Wirth WW 1987. A new Culicoides of the Stigmalis group (Diptera: Ceratopogonidae). Mem Inst Oswaldo Cruz 82: 415-419.

Felippe-Bauer ML, Wirth WW 1988. Culicoides tavaresi, a new species from the state of Rio de Janeiro, Brazil (Diptera: Ceratopogonidae). Mem Inst Oswaldo Cruz 83: 261-263.

Felippe-Bauer ML, Huerta H, Ibañez-Bernal S 2000. A new species of predaceous midge of the genus Monohelea Kieffer from Mexico (Diptera: Ceratopogonidae). Mem Inst Oswaldo Cruz 95: 815-818.

Felippe-Bauer ML, Veras RS, Castellon EG, Moreira NA 2000. A new Culicoides from the Amazonian Region, Brazil (Diptera: Ceratopogonidae). Mem Inst Oswaldo Cruz 95: 35-37.

Ferraz MV 1997. The type specimens of Apoidea (Hymenoptera) deposited in the Entomological Collection of the Instituto Oswaldo Cruz, Rio de Janeiro, RJ, Brazil. Mem Inst Oswaldo Cruz 92: 353-356.

Forattini OP 1956. Nova espécie de Culicoides (Diptera, Ceratopogonidae) de São Vicente, Estado de São Paulo, Brasil. Arq Fac Hig Saude Publica Univ São Paulo 10: 81-84. 
Fox I 1958. Nomenclatorial and bibliographical notes on American blood sucking midges of Culicoides. Proc Tenth Int Congr Ent 1: 137-142.

Goeldi E 1905. Os mosquitos no Pará. Reunião de quatro trabalhos sobre os mosquitos indígenas, principalmente as espécies que molestam o homem. Mem Mus Goeldi Hist Nat Ethnog 4: 1-154.

Gonçalves TCM, Almeida MD, Jurberg J, Lent H 1993. Lista dos exemplares tipos de triatomíneos depositados na Coleção Entomológica do Instituto Oswaldo Cruz, Rio de Janeiro (Hemiptera: Reduviidae). Mem Inst Oswaldo Cruz 88: 327-333.

Hogue CL, Wirth WW 1968. A new Central American sand fly breeding in crab holes (Diptera, Ceratopogonidae). Los Angeles County Museum, Contributions in Science 152: 1-7.

Lane J 1946. Duas Espécies Novas de Ceratopogonídeos Brasileiros [Diptera Ceratopogonidae (Heleidae)], Livro de Homenagem a RF d'Almeida 22: 219-225.

Lutz A 1903. Nota preliminar sobre os insectos sugadores de sangue observados nos Estados de S. Paulo e Rio de Janeiro. Brasil-Médico 29: 281-282.

Lutz A 1912. Contribuição para o estudo das Ceratopogoninas hematofagas encontradas no Brazil. Mem Inst Oswaldo Cruz 4: 1-32.

Lutz A 1913. Contribuicão para o estudo das Ceratopogoninas hematofagas do Brazil. Mem Inst Oswaldo Cruz 5: 45-73.

Lutz A 1914. Contribuicão para o conhecimento das "Ceratopogoninas" do Brasil. Mem Inst Oswaldo Cruz 6: 81-99.

Marchon-Silva V, Lourenço-de-Oliveira R, Almeida MD, Silva-Vasconcelos A, Costa J 1996. The type specimens of mosquitoes (Diptera: Culicidae) deposited in the Entomological Collection of the Instituto Oswaldo Cruz, Rio de Janeiro, Brazil. Mem Inst Oswaldo Cruz 91: 471-478.

Rocha DS, Galvão K, Borgerth SPS, Jurberg J 1998. Lista dos espécimes tipos e de variações genéticas de triatomíneos (Hemiptera: Reduviidae), da Coleção Rodolfo Carcavallo depositados na Coleção Entomológica do Instituto Oswaldo Cruz, Rio de Janeiro, Brasil. Entomol Vect 5: 267-278.

Spinelli GR, Felippe-Bauer ML 1990a. Two new Neotropical species of predaceous midges of the tribe Sphaeromiini (Diptera: Ceratopogonidae). Mem Inst Oswaldo Cruz 85: 87-90.

Spinelli GR, Felippe-Bauer ML 1990b. Sphaerohelea, a new Neotropical predaceous midge genus of the tribe Sphaeromiini (Diptera: Ceratopogonidae). Mem Inst Oswaldo Cruz 85: 195-198.

Spinelli GR, Greiner EC, Wirth WW 1993. The Neotropical bloodsucking midges of the Culicoides guttatus group of the subgenus Hoffmania (Diptera: Ceratopogonidae). Contrib Am Entomol Inst 27: 1-91.

Tavares O, Luna Dias AP 1980. Quatro novas espécies do gênero Culicoides Latreille, 1809, do Estado do Rio de Janeiro, Brasil (Diptera, Ceratopogonidae). Rev Bras Biol 40: 393-399.

Tavares O,. Ruiz RA 1980. Sobre duas espécies novas do gênero Culicoides Latreille, 1809, do Brasil (Diptera, Ceratopogonidae). Mem Inst Oswaldo
Cruz 75: 27-32.

Tavares O, Silva Pereira AJ 1978. Duas espécies novas do genero Monohelea Kieffer, 1917, do Estado do Rio de Janeiro, Brasil (Diptera, Ceratopogonidae). Rev Bras Biol 38: 157-160.

Tavares O, Souza MA de 1978. Sobre três espécies novas do genero Culicoides Latreille, 1809 do Brasil (Diptera, Ceratopogonidae). Rev Bras Biol 38: 619624.

Tavares O, Souza MA de 1979. Sobre algumas espécies do gênero Culicoides Latreille, 1809 do Estado do Rio de Janeiro, Brasil (Diptera, Ceratopogonidae). Rev Bras Biol 39: 611-613.

Tavares O, Souza MA de 1980.Duas espécies novas do gênero Monohelea Kieffer, 1917, do Estado do Rio de Janeiro, Brasil (Diptera, Ceratopogonidae). Rev Bras Biol 40: 95-100.

Wirth WW 1991. Forcipomyia bicolor and related species of the subgenus Lepidohelea in Brazil (Diptera: Ceratopogonidae). Fla Entomol 74: 506-517.

Wirth WW 1992. A new genus of Ceratopogonini (Diptera: Ceratopogonidae) from Brazil. Proc Entomol Soc Wash 94: 276-281.

Wirth WW, Barreto P 1978. New species of Culicoides biting midges (Diptera: Ceratopogonidae). J Med Entomol 14: 553-564.

Wirth WW, Blanton FS 1953a. Studies in Panama Culicoides (Diptera: Heleidae): I, Descriptions of six new species. J Wash Acad Sci 43: 69-77.

Wirth WW, Blanton FS 1953b. Studies in Panama Culicoides (Diptera, Heleidae). III. A new species related to Phlebotomus (Williston). Entomol News 64: 113-120.

Wirth WW, Blanton FS 1955a. Studies in Panama Culicoides (Diptera, Heleidae) IV. Descriptions of three new species. Bull Brooklyn Entomol Soc 50: 100-106.

Wirth WW, Blanton FS 1955b. Studies in Panama Culicoides (Diptera, Heleidae) V. Descriptions of three new species of the subgenus Oecacta Poey. Bull Brooklyn Entomol Soc 50: 121-127.

Wirth WW, Blanton FS 1956a. Studies in Panama Culicoides (Diptera, Heleidae). IX. Two new species related to leoni Barbosa and reevesi Wirth. Bull Brooklyn Entomol Soc 51: 45-52.

Wirth WW, Blanton FS 1956b. Studies in Panama Culicoides VII. The species of the pulicaris and covagarciai group (Diptera, Heleidae). Proc Entomol Soc Wash 58: 211-227.

Wirth WW, Blanton FS 1956c. Studies in Panama Culicoides. VIII. The Neotropical species of the guttatus group of the subgenus Hoffmania (Diptera:Ceratopogonidae). Proc Ent Soc Wash 58: 305-326.

Wirth WW, Blanton FS 1959. Biting midges of the genus Culicoides from Panama (Diptera: Heleidae). Proc US Nat Mus 109: 237-482.

Wirth WW, Blanton FS 1970a. New species of neotropical Culicoides (Diptera: Ceratopogonidae). Fla Entomol 53: 39-45.

Wirth WW, Blanton FS 1970b. A review of the Culicoides nigrigenus group, with two new species (Diptera: 
Ceratopogonidae). Entomol News 81: 141-151.

Wirth WW, Blanton FS 1973. A review of the maruins or biting midges of the genus Culicoides (Diptera: Ceratopogonidae) in the Amazon Basin. Amazoniana 4: 405-470.

Wirth WW, Felippe-Bauer ML 1989. The neotropical biting midges related to Culicoides paraensis (Diptera: Ceratopogonidae). Mem Inst Oswaldo Cruz 84
(Suppl. IV): 551-565.

Wirth WW, Lee DJ 1967. New species of Culicoides from high altitudes in the Colombian Andes (Diptera: Ceratopogonidae). Proc US Nat Mus 124: 1-22.

Wirth WW, Spinelli GR 1992. Immature stages of Forcipomyia seminole Wirth and a related new neotropical species (Diptera: Ceratopogonidae). Fla Entomol 75: 349-356. 
1120 Lista de Ceratopogonidae $•$ ML Felippe-Bauer, SJ de Oliveira 